\title{
Positron Annihilation and Complementary Studies of Stainless Steel Exposed to Sandblasting at Different Angles
}

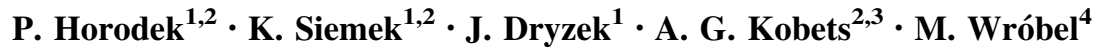

Received: 3 September 2016/Accepted: 3 January 2017/Published online: 12 January 2017

(C) The Author(s) 2017. This article is published with open access at Springerlink.com

\begin{abstract}
Defect studies in subsurface zone in stainless steel 304 AISI samples exposed to sandblasting were performed using positron annihilation spectroscopy techniques. Samples were sandblasted with a different impact angle. Conventional experiments based on positrons emitted directly from the radioactive source allowed us to detect vacancies on the dislocation edges in all samples; however, the total depths of subsurface zones depended strictly on the impact angle, i.e., $35 \mu \mathrm{m}$ for impact angle of $90^{\circ}$ and about $12 \mu \mathrm{m}$ for $30^{\circ}$. The complementary methods such as SEM and optical profilometry revealed also dependencies between the impact angle and roughness of the surface which are not observed in the variable energy positron beam examinations.
\end{abstract}

Keywords Defects $\cdot$ Steel $\cdot$ Sandblasting $\cdot$ Positron spectroscopy

P. Horodek

pawel.horodek@ifj.edu.pl

1 Institute of Nuclear Physics Polish Academy of Sciences, PL-31342 Krakow, Poland

2 Joint Institute for Nuclear Research, Joliot-Curie 6, Dubna 141980, Russia

3 Institute of Electrophysics and Radiation Technologies NAS of Ukraine, Chernyshevsky Str. 28, Kharkiv 61002, Ukraine

4 AGH University of Science and Technology, Mickiewicza Ave. 30, 30-059 Krakow, Poland

\section{Introduction}

Sandblasting is a treatment process involving bombarding the surface with small sand particles. This process is used for cleaning surfaces of metallic products but also for its decoration and improving its texture. However, similarly to other processes of surface treatment such as cutting, sliding and machining, it introduces changes in microstructure on the surface but also below in the subsurface zone (SZ) which is a layer adjacent to the surface. It is characterized by different physical and mechanical properties relative to the specimen interior. SZ represents the region exposed to elastic and plastic deformations introduced below the surface, e.g., as a result of surface treatment process [1]. The studies of SZ are usually performed using conventional engineering methods such as microhardness tests and other analytical techniques like TEM, SEM, XRD [2]. These methods provide wide characteristics; however, plastic deformations occurring during treatment generate structural defects which are largely undetected by the above-mentioned methods. Open-volume defects such as vacancies and their clusters are relatively simple to be observed using positron annihilation spectroscopy (PAS) [3]. Our previous studies have shown successful application of PAS in SZ's studies [4].

The knowledge about impact of sandblasting on SZ's properties is still insufficient. According to the literature, the main direction of investigations is the surface characterization of dental alloys [5]. Another popular trend represents studies of nanocrystallization process caused by blasting and annealing [6-8]. The fact that sandblasting modifies the interior of a sample at a certain depth is rather seldom considered. The PAS studies revealed that this depth is significant as it was reported [3]. 
Within the paper, we report studies of austenitic stainless steel grade 304 AISI exposed to sandblasting, where stream of sand was directed to the surface at various angles. Stainless steel is an important material because sandblasting produces (together with thermal treatment) a nanocrystalline layer improving surface properties [9]. Moreover, metastable austenitic stainless steels exhibiting fcc structure can be transformed to martensitic (bcc) phase by plastic deformation $[10,11]$. In this way, the studies of stainless steel 304 AISI seem to be justified from practical point of view even if results presented in this paper have a basic character.

The main aim of the work is to determine the total thickness of SZ induced by sandblasting using the conventional PAS techniques for this steel. These are based on positrons emitted directly from Na-22 source in the etching experiment. The complementary methods such as the scanning electron microscopy (SEM), optical profilometry and variable energy positron beam (VEP) were also performed to characterize the changes in the surface. In particular, the usefulness of VEP in this topic was tested.

\section{Experimental Details}

\subsection{Sample Preparation}

Stainless steel 304 AISI samples $(0.06 \%$ C, $1.0 \%$ Si, $2.0 \%$ $\mathrm{Mn}, 0.043 \% \mathrm{P}, 0.015 \% \mathrm{~S}, 0.1 \% \mathrm{~N}, 17 \% \mathrm{Cr}$ and $10 \% \mathrm{Ni}$ ) in the form of disks $5 \mathrm{~mm}$ thick and $10 \mathrm{~mm}$ in diameter were used. In the first step, specimens were annealed at $1000{ }^{\circ} \mathrm{C}$ for $4 \mathrm{~h}$ in vacuum conditions of $10^{-5}$ Torr. Then they were slowly cooled down to the room temperature. This procedure allowed us to obtain samples only with residual defects and in the same bulk state. One sample was saved as a reference (RF) while others were exposed to surface treatment. Sandblasting was performed using Renfert Vario Basic Jet blaster. The abrasive material Edelkorund containing $99.8 \%$ aluminum oxide $\left(\mathrm{Al}_{2} \mathrm{O}_{3}\right)$ with size of $50 \mu \mathrm{m}$ was applied. The surfaces were blasted during $60 \mathrm{~s}$ under the pressure of 5 bar with the distance of $10 \mathrm{~mm}$ between the sample and a nozzle. The angle between the surface and the nozzle was equal to $30^{\circ}, 45^{\circ}, 60^{\circ}$ and $90^{\circ}$ for different samples.

\subsection{Experiment Description}

First, surfaces were characterized using Hitachi S-3500 N SEM equipped with EDS Noran 986B-1SPS and by optical profiler WYKO NT9300 (Veeco).

The second group of tests constituted PAS studies. The positron lifetime (LT) measurements were performed using fast-fast spectrometer based on the $\mathrm{BaF}_{2}$ scintillators. The timing resolution equaled $250 \mathrm{ps}$. The isotope $\mathrm{Na}-22$ with activity $32 \mu \mathrm{Ci}$ enveloped into two 7 - $\mu \mathrm{m}$-thick kapton foils was placed between two samples. One sample from each pair was always a reference while the second one was a sandblasted specimen. For that set, LT components were distinguished. The total number of counts in obtained spectra was about $10^{6}$. The deconvolution procedure was carried out by LT code [12] with the positron source correction.

Doppler spectroscopy was realized by the variable energy positron beam facility (VEP) and positrons emitted directly from the $\beta^{+}$source. In the first case, slow positron beam working at Joint Institute for Nuclear Research in Dubna (Russia) was used [13]. The intensity of the flux was $10^{6} \mathrm{e}^{+} / \mathrm{s}$, and the beam spot diameter was about $5 \mathrm{~mm}$. Monoenergetic positrons with incident energy controlled in the range between 0.1 and $36 \mathrm{keV}$ and energy spread $\sim 3 \mathrm{eV}$ were implanted into the sample. The $2.5 \times 10^{5}$ counts in the region of $511 \mathrm{keV}$ peak were registered.

Second type of Doppler broadening of annihilation line (DB) measurements was performed using encapsulated $\mathrm{Na}$ 22 positron source with activity of $15 \mu \mathrm{Ci}$. The isotope was closed in the copper capsule of $5 \mathrm{~mm}$ in diameter and 7 - $\mu$ m-thick titanium window. The exit of positrons was only possible through the window according to this geometry. In this way, the radioactive source was placed in front of the detector with window directed to the top. The sample covered the window with the surface studied. Positrons were implanted in all directions giving information from the sample and the capsule. More details of the measurement setup were given in Ref. [14]. To get the depth characteristics, samples were sequentially etched in paste on the basis of hydrofluoric acid to reduce their thickness. The thickness of the sample was measured using digital microscrew with accuracy $\pm 1 \mu \mathrm{m}$. After etching of about 2- $\mu$ m-thick layer from each side of the sample, the DB measurement was performed. The obtained spectra contained $2 \times 10^{6}$ counts in $511 \mathrm{keV}$ line.

It should be noticed that the average positron implantation depth being the reciprocal value of the linear absorption coefficient [15], it is ca. $29 \mu \mathrm{m}$ for positrons emitted from the Na-22 source in steel. This value indicates the depth from the measured surface which is scanned by positrons in each measurement. About $63 \%$ of emitted positrons get annihilated in such depth.

All DB measurements were performed using HPGe detector with energy resolution of $1.20 \mathrm{keV}$ for energy $511 \mathrm{keV}$. Each obtained spectrum was analyzed to calculate $S$ and $W$ parameters. The first one is given as the ratio of the area below the central part of the annihilation peak to the total area in the range of this line. The $S$ parameter reflects annihilations with low momentum electrons taking place in defects. It is sensitive to open-volume defects like 
vacancies, their cluster or jogs at dislocation lines. Usually, its higher value corresponds to increase in defect concentration. The $W$ parameter in turn is given as the ratio of area below the wing part of $511 \mathrm{keV}$ line to the whole area marked by this line. This parameter represents annihilation with high momentum electrons and is sensitive to the chemical surrounding of the annihilation site. In some cases, the $W$ parameter can point out the changes in the kind or size of the lattice defects.

\section{Results and Discussion}

\subsection{The Results of the Surface Characterization}

It should be noticed at first that during sandblasting we observed the reduction $\Delta d$ of the thickness of the samples, as shown in Table 1 . Decreasing of $\Delta d$ with increasing of the sandblasting angle is clearly visible.

The SEM micrographs of studied samples surfaces are shown on the left in Fig. 1. The reference sample (Fig. 1a) exposes the smooth surface divided by well-marked cracks, which most likely mark grain boundaries. However, after sandblasting surfaces change definitely getting rough, as shown in Fig. 1b-e. SEM investigation does not reveal significant impact of the sandblasting angle on the surface topographies. Similar conclusions can be drawn from optical profiler images presented on the right in Fig. 1. However, some effects were revealed after detailed calculations of quantitative roughness parameters such as $S_{\mathrm{a}}, S_{\mathrm{sk}}$ and $S_{\mathrm{ku}}$ [16]. The obtained values are collected in Table 1 .

The roughness average $\left(S_{\mathrm{a}}\right)$ represents the arithmetic mean of the absolute values of the surface departures from the mean plane. This reflects variations in the height of the surface. Any changes of this parameter come from modification in a manufacturing process. The lowest value is observed for RF. In the case of sandblasted samples, it increases from six to eight times depending on the angle. The higher the angle, the higher the rise in $S_{\mathrm{a}}$.

Skewness $\left(S_{\mathrm{sk}}\right)$ gives the asymmetry of the profile in relation to mean plane. Negative skew points out a

Table 1 Reduction in sample thickness $\Delta d$ and roughness parameter for samples 304 AISI stainless steel exposed to sandblasting at various impact angles

\begin{tabular}{llllc}
\hline Sample (impact angle) & $\Delta d(\mu \mathrm{m})$ & $S_{\mathrm{a}}(\mu \mathrm{m})$ & $S_{\mathrm{sk}}(\mu \mathrm{m})$ & $S_{\mathrm{ku}}(\mu \mathrm{m})$ \\
\hline $\mathrm{RF}$ & - & 0.20 & -2.34 & 65.08 \\
$30^{\circ}$ & 52 & 1.21 & -0.28 & 4.25 \\
$45^{\circ}$ & 31 & 1.33 & -0.51 & 5.00 \\
$60^{\circ}$ & 10 & 1.58 & -0.08 & 4.02 \\
$90^{\circ}$ & 9 & 1.63 & -0.7 & 8.78 \\
\hline
\end{tabular}

predominance of valleys, while positive one is seen on surfaces with peaks. Smooth but covered with particulates surfaces are characterized by positive values. The presence of deep scratches or pits is exhibited by negative $S_{\text {sk }}$. $S_{\text {sk }}$ equaled $-2.34 \mu \mathrm{m}$ for reference sample can be caused by deep grain boundaries produced during annealing. After sandblasting, higher but still negative values of these parameters are noted. Any stronger dependencies between angle and skewness are not observed.

Kurtosis $\left(S_{\mathrm{ku}}\right)$ in turn describes spikiness of the surface or spikes distribution above and below a mean plane. Values $S_{\mathrm{ku}}>3$ are observed for spiky surfaces, $S_{\mathrm{ku}}<3$ are observed for bumpy ones, and perfectly random surfaces have $S_{\mathrm{ku}}=3$. The highest $S_{\mathrm{ku}}$ characterizes RF while for sandblasted specimens this parameter varies from 4.02 to $8.78 \mu \mathrm{m}$. Only for the perpendicular blasting, a notable difference in comparison with smaller angles is visible. In any case, all values are higher than 3 , which indicates a rough surface.

\subsection{VEP Measurements Results}

In Fig. 2a, dependencies of the $S$ parameter values of measured sandblasted samples on the incident positron energy are depicted. There are typical dependencies frequently observed for this kind of material [17]. We can notice that the $S$ parameter values decrease with energy and achieve saturation in case of all samples. The dependency for RF (black circles) stabilizes at the highest positron energy at much lower value of the $S$ parameter. The higher values of the $S$ parameter's saturations observed for sandblasted samples point out the presence of vacancy-type defects trapping positrons. It confirms the significant modification of the subsurface region below the surface by the sandblasting process. One can point out that directly on the surface the values of the $S$ parameter do not differ significantly. It indicates that sandblasting does not create any new positron traps directly at the surface in comparison with the RF sample.

Any important dependencies between the $S$ parameter profiles and sandblasting angle are not reflected. However, for energy lower than $10 \mathrm{keV}$ only for the sample sandblasted at angle $30^{\circ}$, the obtained dependency differs from the other dependencies. Wider analysis of it, e.g., using positron diffusion equation [18] can be inconvenient because of roughness of the surfaces of treated specimens randomly oriented to the beam direction. The mean positron depth, calculated using the well-known formula: $\bar{x}=A E^{n} / \rho$, where $E$ means positron energy in $\mathrm{keV}$, $A=2.62 \mu \mathrm{g} \mathrm{cm}^{-2} \mathrm{keV}^{-\mathrm{n}}, \quad n=1.692$ are Makhov's parameters for iron and $\rho=7.87 \mathrm{~g} / \mathrm{cm}^{3}$ is density of steel [19] is ca. $1.3 \mu \mathrm{m}$ for the maximal positron energy, i.e., $E=35 \mathrm{keV}$. This depth is comparable to the mean 
Fig. 1 SEM micrographs (on the left) and optical profiler images (on the right) for samples 304 AISI stainless steel exposed to sandblasting at various impact angles.

a reference sample and $\mathbf{b}$ e sandblasted ones at $30^{\circ}, 45^{\circ}$, $60^{\circ}$ and $90^{\circ}$, respectively

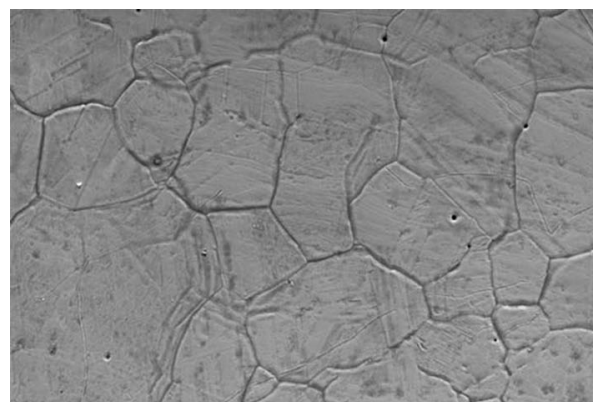

SE wDi6.imi $20.0 \mathrm{kv} \times 500^{\circ}$ iojum
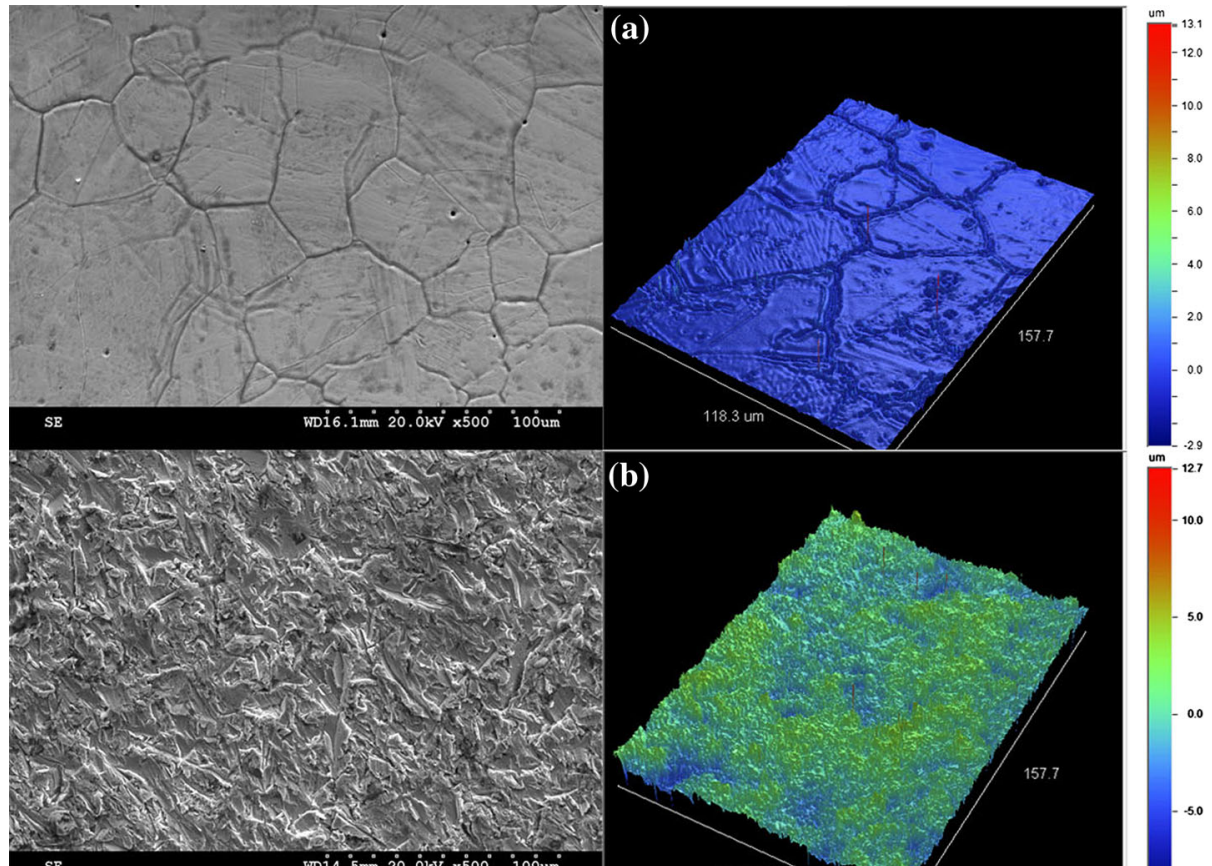

(b)

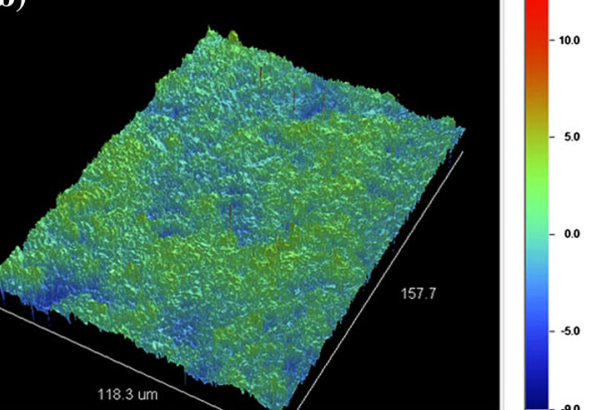

s.t.

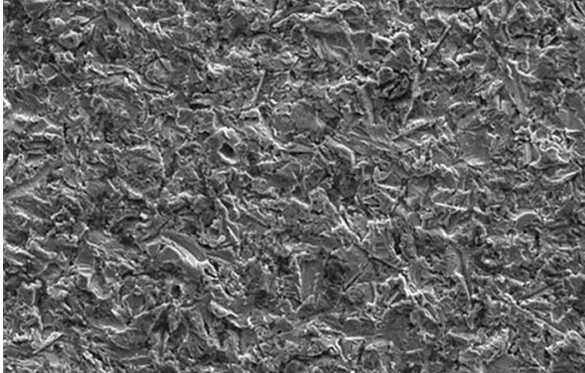

(c)

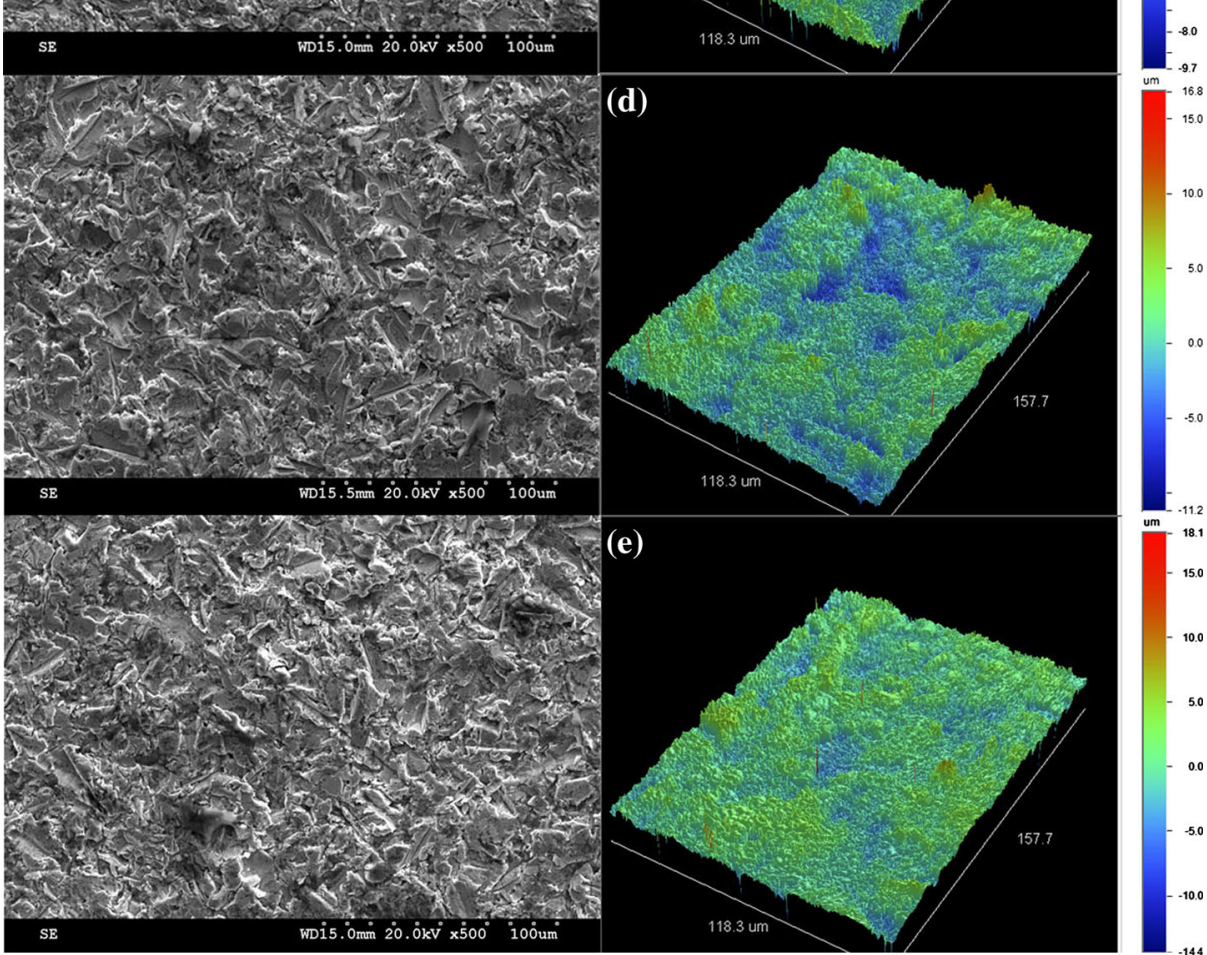


Fig. $2 S$ parameter in dependency on positron energy (on the left) for samples 304 AISI stainless steel exposed to sandblasting at various impact angles. On the right, the $S$ parameter versus the $W$ parameter

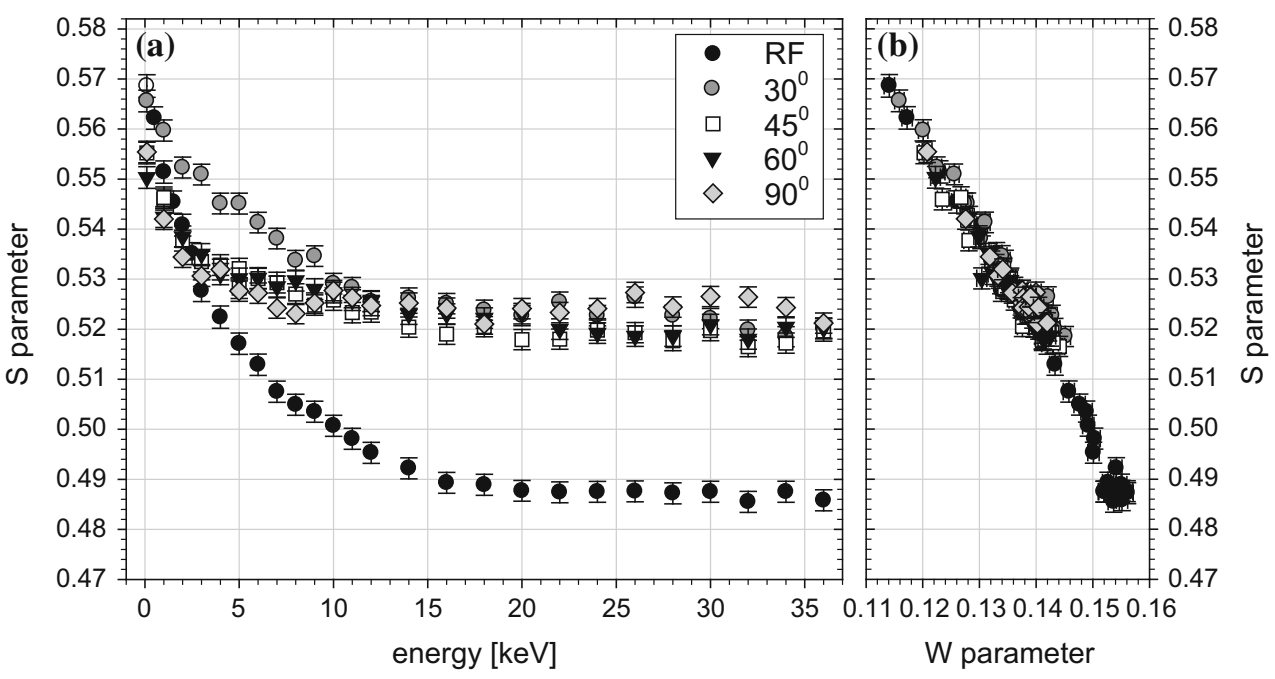

roughness of sandblasted samples, i.e., about $1.5 \mu \mathrm{m}$ presented in Table 1. Thus, one can expect that the positron back-diffusion is disturbed and part of positrons can reach surface earlier than others. The typical solution of the positron diffusion equation does not take into account such a case. However, for the highly deformed surface as it is in the case of the sandblasting the positron diffusion length is very low, about $10 \mathrm{~nm}$; therefore, this effect can be neglected [20].

The linear dependency of $W(S)$ plotted in Fig. 2b informs additionally about the domination of only one kind of vacancy-type defects in all blasted specimens.

\subsection{The Results of the Positron Lifetime Measurements}

The conventional positron lifetime measurements with positrons emitted from the Na-22 source revealed only a single lifetime component, and its values for each of the samples are gathered in Table 2. The value for the RF sample is equal to $106 \pm 1 \mathrm{ps}$; similar value can be found in the literature as a bulk value in iron [4]. The component related to the sandblasted sample can be regarded as mean positron lifetime. Its higher values are observed in all

Table 2 Values of the positron lifetime measured directly on the sandblasted surface for samples 304 AISI stainless steel exposed to sandblasting under various impact angles

\begin{tabular}{ll}
\hline $\begin{array}{l}\text { Sample } \\
\text { (impact angle) }\end{array}$ & $\begin{array}{l}\text { Positron lifetime } \\
(\mathrm{ps})\end{array}$ \\
\hline $\mathrm{RF}$ & $106(1)$ \\
$30^{\circ}$ & $133(1)$ \\
$45^{\circ}$ & $146(1)$ \\
$60^{\circ}$ & $151(1)$ \\
$90^{\circ}$ & $152(1)$ \\
\hline
\end{tabular}

treated samples revealing the existence of structural defects in SZ. The positron lifetimes range between 133 and $152 \mathrm{ps}$, which can be assigned to the positron trapping in vacancies on the dislocation edges. According to Hori et al. [21], defects characterized by positron lifetime between 130 and $175 \mathrm{ps}$ in iron are mainly vacancies bounded by dislocations. This interpretation is also in agreement with results reported by other authors [22-24]. One can notice almost linear dependency between the roughness average $\left(S_{\mathrm{a}}\right)$, as shown in Table 1, and the measured positron lifetime for the samples studied, as shown in Table 2. This can be related that higher roughness results in the increase in surface and subsurface deformation and in higher depth of deformation. This is confirmed in the measurements of the depth profile of the $S$ parameter.

\subsection{The Defect Depth Profile of the SZ}

The depth distribution of defects induced by sandblasting was recognized in the chemical etching experiment, i.e., the sequenced removing of a layer from the treated surface and measurement of the annihilation line shape parameters.

This sequential procedure allowed us to determine the depth profile in respect of the $S$ and $W$ parameters depending on the etched thickness, which is the depth from the surface exposed to sandblasting. The $S$ parameter in the dependency on the etched depth (on the left) and the $W(S)$ plot (on the right) are depicted in Fig. 3.

The hatched region in Fig. 3 a represents the bulk value of the $S$ parameter obtained for RF sample. For this case, the constant level of points is presumed because chemical etching itself does not introduce defects in the interior of the sample, as it was proved in our former studies [14]. In the case of sandblasted samples, the increase in the $S$ parameter close to the surface with increase in the angle is observed. It points out the presence of open-volume defects 

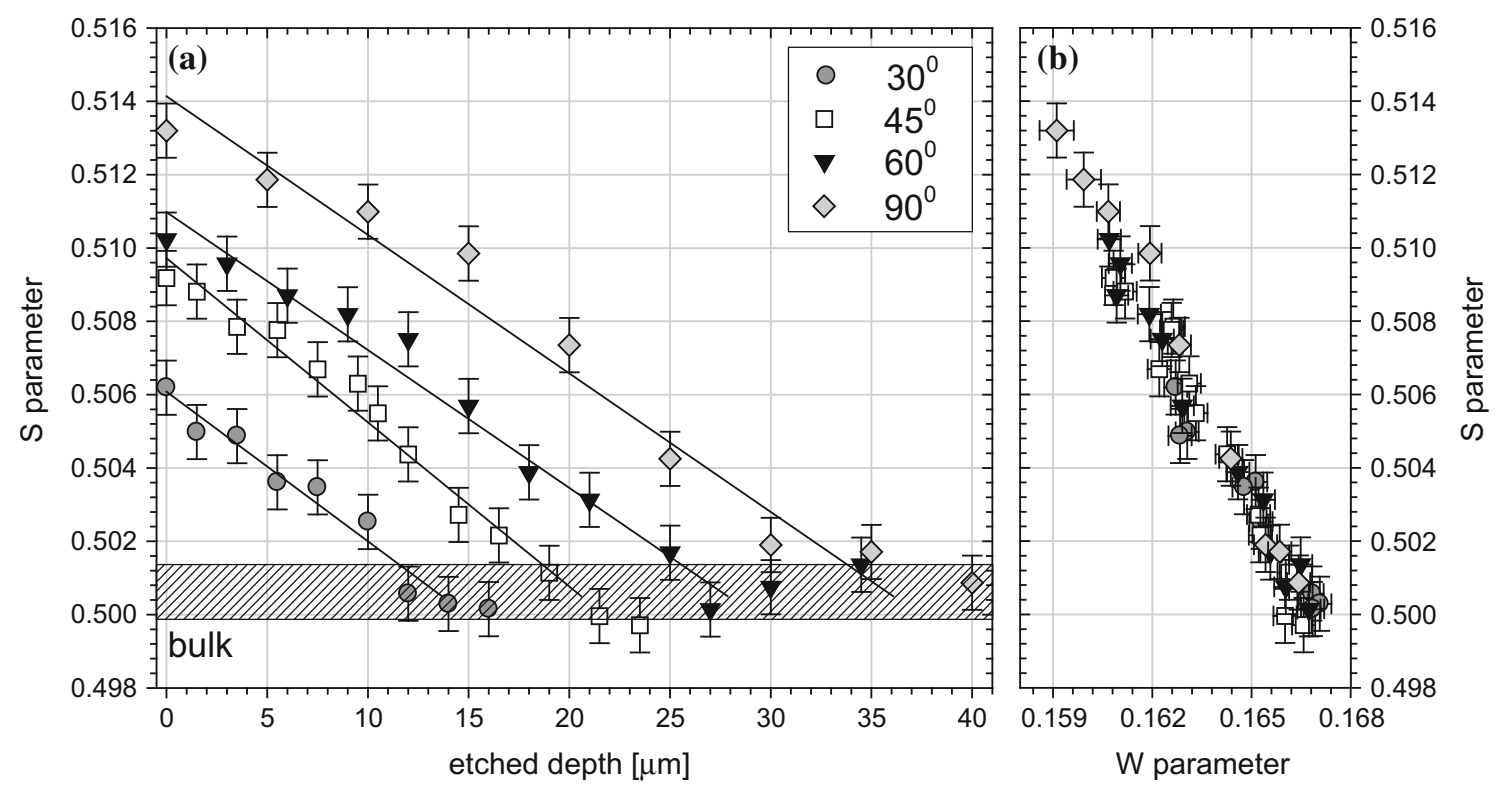

Fig. 3 Dependency of the $S$ parameter on the depth below the surface for the 304 AISI stainless steel samples sandblasted at various impact angles. The hatched region represents the bulk value of the

whose concentration raises with the angle increasing. In each depth profile, the $S$ parameter almost linearly decreases with the depth increase to the bulk value which marks the end of a damaged zone, i.e., SZ. Fitting a linear function to decreasing part of each profile presented in Fig. 3a allows us to approximate the range of defect distribution. It is ca. $12,19,26$ and $35 \mu \mathrm{m}$ for sandblasting at $30^{\circ}, 45^{\circ}, 60^{\circ}$ and $90^{\circ}$, respectively. Another interesting observation is a linear character of the discussed profiles. For comparison, in this kind of stainless steel the exponential decreasing was detected for dry sliding [25]. Also the exponential distribution was observed in sandblasted $\mathrm{Mg}$ [26]. In case of cutting, a clear character of the decay function was hard to evaluate [4].

In Fig. 3b, points of plotted $W(S)$ lie along a straight line pointing out the independence of the size or kind of defect on the impact angle during sandblasting process.

The direct relation between the total depth of the SZ and the angle of the impact of sand stream can be linked to a change in the impact force of sand particles on the surface. Its value is maximal for the perpendicular impact and should decrease with the angle according to the sine function. The dependency of the total depth on the applied load in sliding experiment was observed many times [25]. Another parameter important for the generation of the SZ depending on the angle is the velocity with which sand particles hit the surface.

One should emphasize the effect of etching on the measured values of the $S$ parameter is excluded. As we mentioned above, the implantation range of positrons emitted from the Na-22 source is about $29 \mu \mathrm{m}$ and it is much higher

$S$ parameter obtained for the well-annealed RF sample. On the right, the $S$ parameter versus the $W$ parameter values from the Doppler broadening spectra are depicted

than the average roughness $S_{\mathrm{a}}$ of the surface. Additionally, as we checked experimentally $S_{\mathrm{a}}$ values slightly increase to ca. $8 \mu \mathrm{m}$ with the increase in the etched depth.

\section{Conclusions}

Sandblasting as a surface treatment process causes the increase in the surface roughness and modifies the subsurface zone by creation of a significant number of the open-volume defects. It was found that the roughness of the sandblasted surface $\left(S_{\mathrm{a}}\right)$ increases with the impact angle. The measurements performed using positrons emitted directly from the $\mathrm{Na}-22$ source revealed the presence of vacancies on dislocation lines. The total thickness of subsurface zone increases with the angle from $12 \mu \mathrm{m}$ for $30^{\circ}$ up to $35 \mu \mathrm{m}$ for treating perpendicularly to the surface. Positron beam tests seem to be not applicable to investigated samples.

Open Access This article is distributed under the terms of the Creative Commons Attribution 4.0 International License (http://crea tivecommons.org/licenses/by/4.0/), which permits unrestricted use, distribution, and reproduction in any medium, provided you give appropriate credit to the original author(s) and the source, provide a link to the Creative Commons license, and indicate if changes were made.

\section{References}

1. Rice, S., Nowotny, H., Wayle, S.: A survey of the development of subsurface zones in the wear of materials. Key Eng. Mater. 33, 77-100 (1989) 
2. Zandrahimi, M., Reza Bateni, M., Poladi, A., Szpunar, J.: The formation of martensite during wear of AISI 304 stainless steel. Wear 263, 674-678 (1997)

3. Dryzek, E.: Defect depth profiling after sphere indentation and blasting in aluminium and aluminium alloy detected by positron annihilation. J. Mater. Sci. 38, 3755-3763 (2003)

4. Horodek, P., Dryzek, J., Wróbel, M.: Positron annihilation study of defects induced by various cutting methods in stainless steel grade 304. Trobol. Lett. 45, 341-347 (2012)

5. Su, N., Yue, L., Liao, Y., Liu, W., Zhang, H., Li, X., Wang, H., Shen, J.: The effect of various sandblasting conditions on surface changes of dental zirconia and shear bond strength between zirconia core and indirect composite resin. J. Adv. Prosthodont. 7, 214-223 (2015)

6. Belbasi, M.R., Ramezani, M.: The effect of sandblasting time on producing nanocrystalline surface of PO733 steel (DIN: 1.8509). Mater. Phys. Mech. 17, 33-37 (2013)

7. Li, X.Y., Wang, D.Y.: Mechanical, electrochemical and tribological properties of nano-crystalline surface of 304 stainless steel. Wear 255, 836-845 (2003)

8. Fard, S.B., Guagliano, M.: Effects of surfaces nanocrystallization induced by shot peening on material properties: a review. Frattura Integr. Strutt. 7, 3-16 (2009)

9. Wang, X.Y., Li, D.Y.: Mechanical, electrochemical and tribological properties of nano-crystalline surface of 304 stainless steel. Wear 255(7-12), 836-845 (2003)

10. Bendel, L.P., Shellock, F.G., Steckel, M.: The effect of mechanical deformation on magnetic properties and MRI artifacts of type 304 and type 316L stainless steel. J. Magn. Reson. Imaging 7(6), 1170-1173 (1997)

11. Multigner, M., Frutos, E., González-Carrasco, J.L., Jiménez, J.A., Marín, P., Ibáñez, J.: Influence of the sandblasting on the subsurface microstructure of 316LVM stainless steel: implications on the magnetic and mechanical properties. Mater. Sci. Eng. C 29, 1357-1360 (2009)

12. Kansy, J.: Microcomputer program for analysis of positron annihilation lifetime spectra. Nucl. Instrum. Methods Phys. Res. A 374, 235-244 (1996)

13. Horodek, P., Kobets, A.G., Meshkov, I.N., Sidorin, A.A., Orlov, O.S.: Slow positron beam at the JINR, Dubna. Nukleonika 60(4), 725-728 (2015)

14. Horodek, P., Dryzek, J.: Application of encapsulated 22-Na isotope to Doppler spectroscopy based on positrons emitted directly from the source. Acta Phys. Pol. B Proc. Suppl. 9(2), 1001-1007 (2016)

15. Dryzek, J., Singleton, D.: Implantation profile and linear absorption coefficients for positrons injected in solids from radioactive sources $22 \mathrm{Na}$ and $68 \mathrm{Ge} 668 \mathrm{Ga}$. Nucl. Instrum. Methods Phys. Res. B 252, 197-204 (2006)

16. Bhushan, B.: Introduction to Tribology. Wiley, New York (2002)

17. Wu, Y.C., Zhang, R., Chen, H., Li, Y., Zhang, J., Zhu, D.M., Jean, Y.C.: Corrosion of iron and stainless steels studied using slow positron beam technique. Radiat. Phys. Chem. 68, 599-603 (2003)

18. Dryzek, J., Horodek, P.: The solution of the positron diffusion trapping model tested for profiling of defects induced by proton implanted in stainless steel. Appl. Phys. A 121, 289-295 (2015)

19. Horodek, P., Dryzek, J.: GEANT4 simulation of slow positron beam implantation profiles. Nucl. Instrum. Methods Phys. Res. B 266, 4000-4009 (2008)

20. Schultz, P.J., Lynn, K.G.: Interaction of positron beams with surfaces, thin films, and interfaces. Rev. Mod. Phys. 60, 701-781 (1988)

21. Hori, K., Koike, K., Oshima, R.: Investigation of lattice defects in the early stage of fatigue in iron by positron annihilation techniques. Appl. Surf. Sci. 242, 304-312 (2005)

22. Hidalgo, C., Gonzalez-Doncel, G., Linderoth, S., San Juan, J.: Structure of dislocations in $\mathrm{Al}$ and $\mathrm{Fe}$ as studied by positronannihilation spectroscopy. Phys. Rev. B 45(13), 7017-7021 (1992)

23. Mostafa, K.M., De Baerdemaeker, J., Calvillo, P.R., Van Caenegem, N., Houbaert, Y., Segers, D.A.: Study of defects in iron based alloys by positron annihilation techniques. Acta Phys. Pol. A 113(5), 1471-1478 (2008)

24. Lee, J.L., Waber, J.T.: Positron annihilation study of dislocations produced by polishing in the surface of iron single crystals: part I. Density profile and removal by annealing. Metall. Trans. A 21(7), 2037-2045 (1990)

25. Dryzek, J., Horodek, P., Wróbel, M.: Use of positron annihilation measurements to detect the defect beneath worn surface of stainless steel $1.4301(\mathrm{EN})$ under dry sliding condition. Wear 295, 264-269 (2012)

26. Dryzek, J., Dryzek, E., Suzuki, T., Yu, R.: Subsurface zone in pure magnesium studied by positron lifetime spectroscopy. Tribol. Lett. 20(1), 91-97 (2005) 